\title{
Cognitive biases in developing biased Artificial Intelligence recruitment system
}

\author{
Melika Soleimani \\ School of Management \\ Massey University \\ $\underline{\text { m.soleimani@massey.ac.nz }}$
}

\author{
Ali Intezari \\ UQ Business School \\ University of Queensland \\ a.intezari@business.uq.edu.au
}

David Pauleen

School of Management

Massey University

d.pauleen@massey.ac.nz

\author{
Nazim Taskin \\ Management Information Systems \\ Department \\ Bogazici University \\ nazim.taskin@boun.edu.tr
}

\begin{abstract}
Artificial Intelligence (AI) in a business context is designed to provide organizations with valuable insight into decision-making and planning. Although AI can help managers make decisions, it may pose unprecedented issues, such as datasets and implicit biases built into algorithms. To assist managers with making unbiased effective decisions, AI needs to be unbiased too. Therefore, it is important to identify biases that may arise in the design and use of AI. One of the areas where AI is increasingly used is the Human Resources recruitment process. This article reports on the preliminary findings of an empirical study answering the question: how do cognitive biases arise in AI? We propose a model to determine people's role in developing AI recruitment systems. Identifying the sources of cognitive biases can provide insight into how to develop unbiased AI. The academic and practical implications of the study are discussed.
\end{abstract}

\section{Introduction}

With the rapid development of technologies, businesses have been placed in new and different competitive situations [2]. To gain an edge, organizations attempt to progress towards optimal decision-making [11]. To make optimal decisions, managers are increasingly recognizing the importance of decision support tools and techniques such as Decision Support Systems (DSS), Business Intelligence (BI), and Artificial Intelligence (AI) in decision-making process [8], [34].
$\mathrm{AI}$ is considered to be one of the prominent analytics technologies to collect and process data [2]. The ubiquity of data requires the growth of analytics in managerial decision-making. Analytics is the technique for examining data to uncover hidden patterns, correlations and other insights through using statistical and operations analyzes [21].

AI finds answers to a question or solutions to a problem based on what algorithms tell the machine to do [2]. Algorithms consist of a sequence of computational steps that transform inputs into outputs [12]. Collected and processed data are used as inputs of algorithms and knowledge is extracted through machine learning algorithms [10]. Although AI can assist in decision-making [2], the datasets and algorithms that guide AI can be influenced by human biases [43].

Research on AI-assisted decision-making shows that biases are one of the challenges of developing AI [31], [39], [41], [25]. Cognitive biases in decision-making represent an individual's deviations from rational judgement and decisions [17].

In this article, we offer preliminary findings to the question: how do cognitive biases arise in AI? To answer the research question, we are using a grounded theory research design. We are particularly interested in understanding how the interaction and cooperation between managers and AI developers in the development process of AI lead to biased AI. We propose a model of the manager-developer interaction in developing $\mathrm{AI}$ in relation to recruitment processes.

In the sections below, a definition of AI along with information on AI development are provided. Then, cognitive biases and cognitive biases in developing AI are explained. Next, data collection and analysis are 
described. Then, based on the preliminary findings, HR managers' and developers' roles in developing biased $\mathrm{AI}$ are further discussed.

\section{Theoretical Background}

\subsection{Artificial Intelligence}

The term AI was coined by John McCarthy in 1956. However, work on AI was started during the Second World War by Alan Turing, an English mathematician and computer scientist [35]. The collaboration of scholars, such as Herbert Simon, Allen Newell, Claude Shannon, Nathaniel Rochester, together with research by Marvin Minsky at the Massachusetts Institute of Technology and John McCarthy of Stanford, developed early computer models of human cognition [33], [8].

By considering human intelligence in his definition, Minsky defines AI as "the science of making machines do things that would require intelligence if done by men" [32, p.v.]. Kaplan and Haenlein [26] extend the approach of these scientists' such as Newell, Shannon, and Rochester by specifically focusing on how AI can do tasks that require human intelligence. They define AI as "a system's ability to interpret external data correctly, to learn from such data, and to use those learnings to achieve specific goals and tasks through flexible adaptation" [26, p.17].

The learning ability which takes place by extracting information from data through Machine Learning (ML) algorithms, is the distinctive characteristic of AI [1] compared to other forms of technology. Although AI and ML are often used interchangeably [7], they are different concepts. ML is a central part of AI and provides systems the ability to learn and change when exposed to new data without being explicitly programmed [26].

ML uses mathematical models [12] and statistics to prepare training data and derive a set of results from datasets [28]. Machine learning is intertwined with statistics to derive a set of results from data sets [28].

After an algorithm has been trained by applying a subset of historical data, it can process new data. "The goal of training is to develop a model capable of formulating a target value (attribute), some unknown value of each data object" [2, p.22]. Outputs of the building model can be reused to retrain the AI model and achieve better performance [44]. Figure 1 illustrates the process of developing ML.

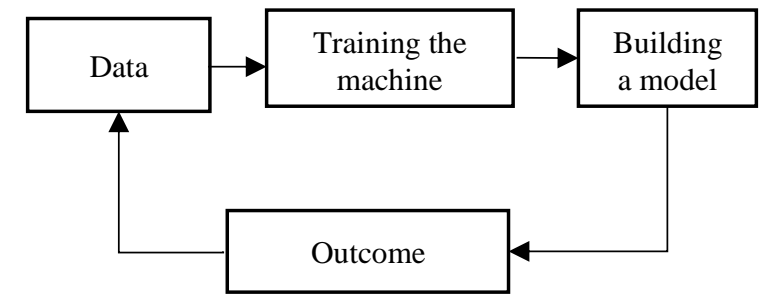

Figure 1- The process of developing Machine Learning and producing an outcome

For example, to predict whether an employee will stay or leave within the next year the inputs of the algorithms are the historical data which tell developers who did and did not leave within the last year. The outcomes would be predicting turnover for the next year. Through deriving a model based on the historical data, predictions on future data are made [2].

To develop AI for managerial decision-making, huge amounts of data need to be collected from numerous sources [23]. The data can come from either internal or external sources, or both [2]. Internal data sources include data warehouses and enterprise resource planning applications [2], which generate and hold data within an organization. If there is a lack of data to train algorithms, datasets from external sources, such as public sources can be used [2]. Any forms of data including images, texts, transactions, human and environmental sensing data ('big data') [20] can be applied as training datasets.

Using external datasets may pose some challenges due to issues such as the lack of detailed and accurate information in datasets [22]. Therefore, the need for using numerous internal and external data sources, requires developers to prepare a consistent dataset by joining up internal and external datasets.

After data preparation, developers develop machine learning models and train them with training datasets [23]. To formulate algorithms, factors and their weights [31] and managers' assumptions should be analyzed and built into the model [41]. Moreover, developers must determine relevant variables as indicators among datasets and clear up ambiguities in chosen indicators [23].

To answer the question of how cognitive biases arise in $\mathrm{AI}$, the individual roles of managers and developers should be considered. In the following section, cognitive biases and cognitive biases in developing $\mathrm{AI}$ is discussed. 


\subsection{Cognitive biases}

Cognitive biases refer to a systematic error in thinking or reasoning [29]. Cognitive biases are the result of using shortcuts in thinking, also termed heuristics [6]. In a significant number of decisions, the decision quality is affected by cognition [3]. Scholars, including Barnes [5], Duhaime and Schwenk [15], and Schwenk [37], have proposed that the biases identified in laboratory context may be seen in decision making. Also, cognitive biases may appear in designing and programming AI and providing datasets for machine learning.

\subsection{Cognitive biases in developing AI}

Cognitive biases may exist in AI systems from the beginning of the development process [25] in datasets as inputs for training algorithms [39]. The design of AI, the same as other software systems, incorporate the ideas of the end-users such as managers [41]. AI is designed to aid management decision-making. However, biases may be transmitted to the AI being designed due to biased assumptions. When developers cannot formulate managers' assumptions objectively or do not choose the best sets of variables from datasets to train algorithms [41] biased algorithms can be developed.

An example of biased datasets that affect decisionmaking by AI is Amazon's AI Recruiter that made biased decisions against women [25]. Amazon AI preferred male candidates while sorting through candidates to choose promising candidates. Kaplan and Haenlein [25] state that this example shows if a firm has a bias against some group of applicants, ML will learn the bias from past decisions and will perpetuate the same bias.

This paper seeks to explain how AI recruitment systems subsume HR managers' and developers' cognitive biases. In the following sections, the research design is explained. Findings are discussed and a model is conceptualized that illustrates HR managers' and developers' roles in developing biased AI based on our preliminary findings.

\section{Research Design}

This study examines how HR managers' and developers' cognitive biases in the recruitment and selection process lead to developing biased AI. To conduct this research an interpretive exploratory study using classic Grounded Theory is undertaken [18]. A semi-structured interview is used as the data collecting method.
HR managers from different sectors in New Zealand and Australia who have more than three years of experience in recruitment are being interviewed. Informants' familiarity with AI varied ranging from limited knowledge of AI applications to conceptually and technically knowledgeable about AI. Four participants were male and six were female.

The informants are being asked open and broad questions to talk freely about the subject, including "What happens during a typical recruitment and hiring process", "Have you ever seen a hiring decision that now you think was a bad decision (the right candidate was not chosen)"; "How has the process changed in recent years with the introduction of technologies such as AI"; "Do you think AI can improve the quality of the recruitment process? if so, how?"; "How do you think the interaction between HR managers and AI can make both parties better over time in the recruitment and selection process?".

\subsection{Data analysis}

The process of data collection and data analysis took place at the same time. To identify categories of the collected data, three coding strategies - open coding, axial coding, and selective coding were used [19]. Categories had been identified and presented using QSR NVivo 12 software package.

The core category represents two sub-core categories: HR managers roles and AI developers' roles. The sub-core categories are based on four conceptual categories that emerged during axial coding. Table 1 shows the samples of preliminary findings.

Table 1- Samples of preliminary findings 


\begin{tabular}{|c|c|c|c|c|}
\hline \multicolumn{2}{|c|}{$\begin{array}{c}\text { Selective } \\
\text { Coding }\end{array}$} & \multirow{2}{*}{$\begin{array}{c}\text { Axial } \\
\text { Coding }\end{array}$} & \multirow{2}{*}{ 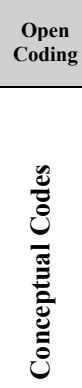 } & \\
\hline ن. & 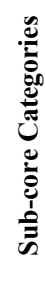 & & & Exemplary Excerpts \\
\hline \multirow{4}{*}{ 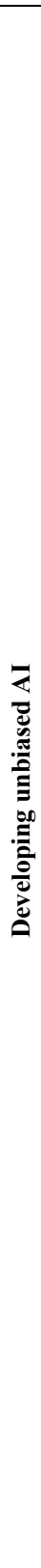 } & \multirow{4}{*}{ 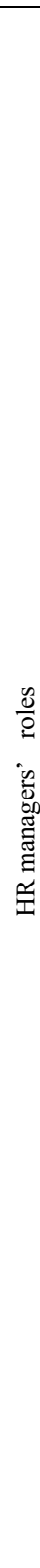 } & \multirow{2}{*}{ 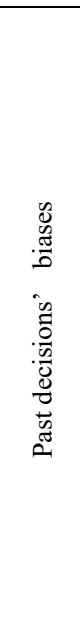 } & 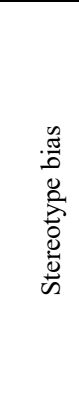 & $\begin{array}{l}\text { "So, I know the engineers } \\
\text { at that company are } \\
\text { really good. And so that } \\
\text { person gets a little bit } \\
\text { more credibility when I } \\
\text { read her CV because I } \\
\text { know from experience } \\
\text { that she's a really good } \\
\text { engineer if she's been } \\
\text { working for five years in } \\
\text { that company" } \\
\text { (Informant 10). }\end{array}$ \\
\hline & & & 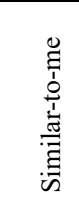 & $\begin{array}{l}\text { "people in the panel are } \\
\text { somehow similar. Yeah, } \\
\text { so they kind of reinforces } \\
\text { the, you know, hiring } \\
\text { someone like us" } \\
\text { (Informant 4). }\end{array}$ \\
\hline & & \multirow{2}{*}{ 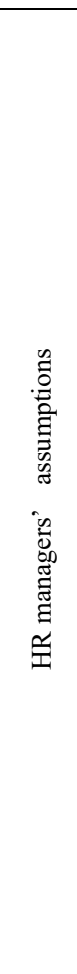 } & 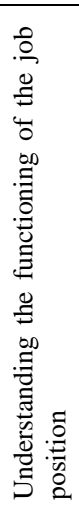 & $\begin{array}{l}\text { "Maybe don't know what } \\
\text { they're looking for. And } \\
\text { so I think what we need to } \\
\text { do before AI can really } \\
\text { help us fix the start of the } \\
\text { process in terms of } \\
\text { understanding what it is } \\
\text { that we're looking for } \\
\text { first, so that everyone } \\
\text { knows, everyone at the } \\
\text { start of the process is } \\
\text { aware of what we're } \\
\text { looking for and drawing } \\
\text { the right information } \\
\text { out"(Informant 8). }\end{array}$ \\
\hline & & & 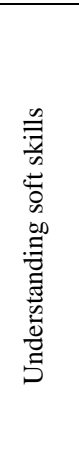 & $\begin{array}{l}\text { "You need to draw out } \\
\text { their personality. And so } \\
\text { you need to make them } \\
\text { comfortable and have to } \\
\text { be able to give you their } \\
\text { personality and } \\
\text { understand. And so you } \\
\text { need to draw that } \\
\text { information out at the } \\
\text { start, you need to make a } \\
\text { very open-ended, you } \\
\text { know, it's just } \\
\text { questioning" (Informant } \\
\text { 5). }\end{array}$ \\
\hline
\end{tabular}

\begin{tabular}{|c|c|c|c|}
\hline \multirow{2}{*}{ 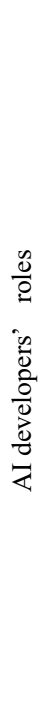 } & 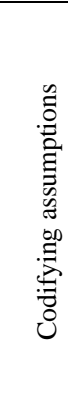 & 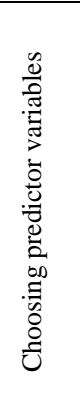 & $\begin{array}{l}\text { "It also depends on } \\
\text { managers, so you might } \\
\text { have a role and over a } \\
\text { period of } 20 \text { years is five } \\
\text { different managers that } \\
\text { manage that role, the } \\
\text { chosen elements will be } \\
\text { very different for all five } \\
\text { of those because the } \\
\text { managers will put their } \\
\text { own view" (Informant } \\
\text { 10). }\end{array}$ \\
\hline & 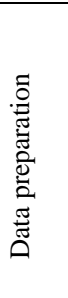 & 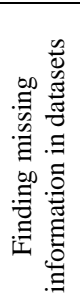 & $\begin{array}{l}\text { "So, HR hasn't been very } \\
\text { data-driven. and it's } \\
\text { insights. A lot of the } \\
\text { transactional data that } \\
\text { they look at is more } \\
\text { around leave and pay and } \\
\text { some of those kinds of } \\
\text { logistical sides of HR" } \\
\text { (Informant 7). }\end{array}$ \\
\hline
\end{tabular}

In the following sections, the conceptual categories and conceptual codes are discussed and some informants' quotes that represent the categories are provided.

\section{Findings}

The preliminary findings of this study elucidate cognitive biases in the recruitment and selection process that may arise in datasets for training ML models. Moreover, the factors that may lead to developing biased AI are discussed.

Our participants were consistent about two biases, similar-to-me and stereotype bias that they believe to be very likely to happen in the design of AI for the recruitment and selection process.

"Just a connection that person is just like me I could work with them. They're going to be easy. They've got a nice smile. They're going to be great with the customers" (informant 2).

"Favoritism of what school they've been to, there's also a favoritism of companies they have been working (informant 3)".

Based on the preliminary findings, inappropriate HR managers' assumptions lead to biased decisions. Inappropriate assumptions happen due to a weak understanding of the functioning of a job, and inappropriate understanding of required soft skills for a job position.

"People do not necessarily understand what they exactly want, people retrofitting a role around rather than considering what the role is and what they need 
first. To get your applicants quicker you really need to understand what it is you're looking for? what skills? What age what stage is going to be relevant for this" (informant 7).

Similarly, another statement shows that how a manager's assumptions may lead to biased decisions even when there is no fact to support the decision.

"I rejected a candidate by saying that he is not extrovert. Actually, that was my gut feeling and there was nothing that really tested the skill and being extrovert was not needed for the role but all team members were kind of extrovert" (informant 8).

Our preliminary findings reveal that there might be some missing information in datasets. Informants believe that they may not have all information on the recruitment and selection process and it may cause some issues for developers to fill out the missing information.

"We don't feed through, like total information into data so they're not getting everybody's information" (informant 6).

"if you improve the quality of data feeding into the machine learning, that would give that process the better ability to help you select the best performer" (informant 4).

Furthermore, developers might not be able to choose the optimal sets of variables. Informants believe that managers are not consistent in defining relevant criteria to choose the best candidates for a role. Therefore, it is difficult to develop a robust AI system.

"AI can be very effective if the organization can define what great looks like, and actually, it's a lot harder than you think. You see to a manager what makes a great retail sales rep and telecommunications is not the same as others. Across all of the retail managers, you get a different answer. so long as you can build a, you know, a robust tool that you're confident matches the criteria that great performance looks like, then it's incredibly effective" (informant 4).

\section{Discussion}

According to the preliminary findings, ML models could be trained with historical datasets that include cognitive biases such as similar-to-me biases and stereotypes. Similar-to-me bias refers to possessing similar demographics such as age, race, and attitudinal characteristics to the recruiter [38]. A stereotype bias is defined as "a fixed, over-generalized belief about a particular group or class of people" [9, p.227]. Also, informants believe that in the recruitment context, managers' assumptions that cause problems in formulating algorithms include inappropriate understanding of the functioning of the job position and required soft skills.

Based on our findings, developers may have missing information in datasets. Developers may rely on inadequate information in datasets that might lead to biases in datasets while doing data preparation. Moreover, managers may not be consistent in choosing optimal criteria to recruit candidates for a role. Therefore, it is difficult for developers to choose the optimal sets of variables to formulate algorithms.

Figure 2 illustrates a conceptual model of HR managers' and developers' roles in developing biased AI based on our preliminary findings.

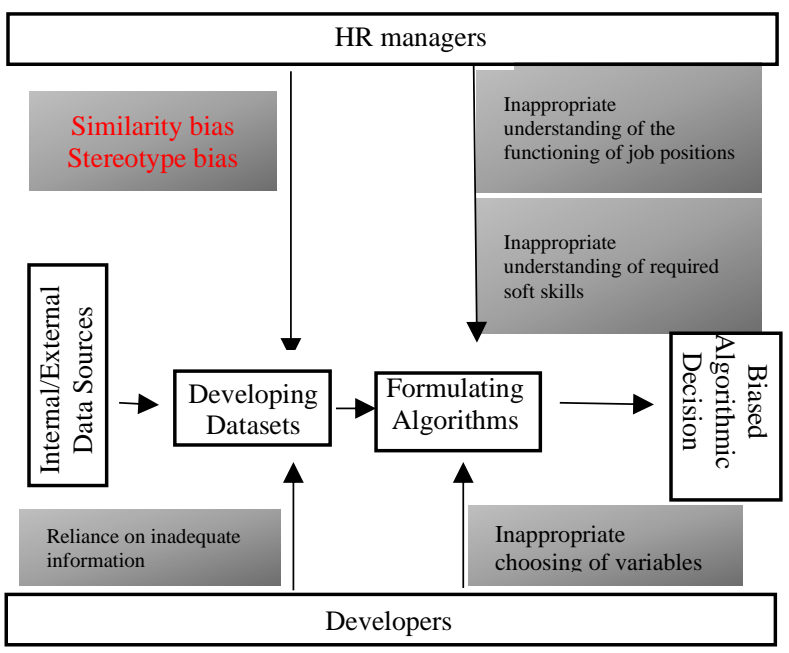

Figure 2- HR managers' and developers' roles in developing biased AI

The model draws upon the concepts for the development process of AI. Datasets as inputs and formulating algorithms are the central aspects of developing AI according to "algorithms as producing answer "model by Kirsten Martin [31, p.837]. The model shows that the two main components of developing AI, datasets and algorithms may be biased due to four reasons.

\section{Managers' biases in datasets}

Managers' decisions are used as datasets to train AI systems. Managers' decisions may be imperfect due to their biases [24]. Although some sensitive variables such as gender, race, or sexual orientation may be 
eliminated from datasets, the reflection of historical or social inequities may affect datasets [30]. For example, biases such as "taste-based discrimination, or poorly calibrated statistical discrimination" [13, p.2] can be found in datasets as inputs of AI systems. The two biases found in our findings, similar-to-me and stereotype bias, are highlighted in red in the conceptual model.

\section{Managers' biases in formulating algorithms}

To apply AI in managerial decision-making such as human resource management, the managers' assumptions are built into algorithms [41]. To develop the algorithms, organizations must articulate their decision-making process and which criteria they choose [41]. AI based decision-making requires specific objective functions.

However, coding objective functions based on decision-making by humans is difficult [39] as in some cases managers cannot answer questions as to why decisions were made even when the results are good [16]. Human decision-makers sometimes make decisions intuitively based on "a set of tacitly held preferences" [39, p.3].

Shrestha, Ben-Menahem and Krogh [39] further explain that humans cannot consider the set of factors separately and explicitly explain which criteria are important and with what weight. For example, a manager making a recruitment decision about how a candidate fits the organizational culture may not be able to explicitly explain which criteria are important and how they are weighted. Based on our findings, HR managers' inappropriate understanding of the functioning of the job positions and required soft skills that may lead to arising biases in formulating algorithms are added to the conceptual model.

\section{Developers' biases in data preparation}

Given that solely relying on one data source, such as managers' past decisions, may lead to anchoring bias [16], more diverse data sets need to be used in training ML algorithms. In ML, different samples of datasets should be selected for training, validating and testing the model from different periods to avoid status quo bias [41], [16].

When the training dataset is not enough, external datasets from different sources such as social media, mobile application and publicly available data can be used [14]. Although using external data sources increase datasets, it may increase biases due to inaccurate and inconsistent outcomes of the external datasets [22].

In organizations, datasets exist for different purposes. The different purposes affect which data are collected and the details of the collected data [4]. Before utilizing datasets, data preparation is required. Preparing datasets that have already been collected for different purposes in organizations is time-consuming [16]. Data cleaning is part of data preparation and it is a step related to data collection and storage [40].

One way of cleaning data is filling out missing values in datasets. There are different ways to fill out missing values in datasets such as single value imputation and model-based imputation. Single value imputation can be considered as using mean and median or the last observation carried forward. Using the last observation means that the last observation has not been changed which is unrealistic [36] and can be biased. For example, the missing information about the future performance of the job applicants can be predicted by considering the performance of similar job applicants who have been hired before [27].

Also, by using model-based imputation such as linear regression, the correlation between variables will be overestimated because the uncertainty in the missing data is not taken into account [36]. In the same manner, informants in our study explain that there is some missing information in datasets which affect the quality of data used as inputs for algorithms.

\section{Developers' biases in formulating algorithms}

To develop the algorithms, first developers formalize the end users' assumptions [41]. According to Taniguchi, Sato, and Shirakawa [42], biases related to inappropriate assumptions of correlations such as symmetric and mutually exclusive bias may happen while the assumptions are formulated.

Symmetric bias is the result of inferring "if q then $p$ " after knowing that "if $p$ then $q$ ". For example, from this conditional statement "if the weather was rainy then the ground was wet" someone may infer that when the ground was wet then the weather was rainy. Also, mutually exclusive bias concluding that "if $\sim p$ then $\sim q$ " is correct after convincing that "if $p$ then q". For example, interpreting this conditional statement "[i]f you do not clean up your room, you will not be allowed to play" as "if I clean up my room, then I am allowed to play" is a mutually exclusive bias [42].

To formalize the assumptions, the optimal datasets of variables should be chosen. The choice of variables as inputs of algorithms can be biased [27], [16]. Choosing the small sets of variables [13], i.e., the reduced representative of variables [27] underperforms the outcomes of the algorithms.

According to Tambe, Cappelli, and Yakubovich, [41], selecting the variables makes it more difficult to realize the real effects. This bias in data science is called "collider effect" (p. 29). For instance, algorithms in 
human resource management cannot understand any attributes that differentiate the best performers from bad performers by considering dependent variables only from the best performers.

Kleinberg, Ludwig, Mullainathan, Sunstein, [27] argue that to develop the training algorithms, developers choose among the available predictors to include in the statistical models. Predictors in the final prediction model are determined by the data through using the chosen predictors. If there are multiple correlated predictors in a dataset, the training algorithms cannot detect the causal inference.

Kleinberg, et al., [27] further explain that, for example, in the recruitment decision-making if an attribute such as race is not included in the model, maybe a correlated proxy of a race like the neighbourhood of residence and high school attended might be entered in the algorithms. Therefore, while race is not chosen among the subsets of variables, it does not mean that correlated proxy is not playing a role.

To interpret the behaviour of variables and assess the performance of the algorithms in the business context, the most appropriate metrics should be determined [41], [16]. If only one metric always is chosen, say arithmetic mean rather than considering the other metrics such as geometric mean, mode, and median, bias can appear from the lack of analysis of the distribution of the variables [16].

Based on our preliminary findings, choosing optimal sets of variables is difficult as managers may not agree upon optimal criteria to hire the best candidates. As it is shown in the conceptual model, inappropriately choosing of optimal sets of variables causes problems for formulating algorithms.

Table 2, summarizes the four factors of developing biased AI based on managers' and developers' roles in two central aspects of developing $\mathrm{AI}$; datasets and algorithms.

Table 2- Managers' and AI developers' roles in developing biased AI

\begin{tabular}{|c|c|c|}
\hline 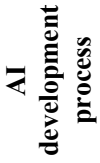 & $\begin{array}{l}\text { Managers' roles in } \\
\text { developing biased AI }\end{array}$ & $\begin{array}{l}\text { AI developers' roles in } \\
\text { developing biased } \mathrm{AI}\end{array}$ \\
\hline 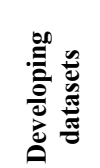 & $\begin{array}{l}\text { Imperfect past } \\
\text { decisions as internal } \\
\text { datasets due to } \\
\text { similar-to-me and } \\
\text { stereotype biases }\end{array}$ & $\begin{array}{l}\text { Reliance on } \\
\text { inadequate } \\
\text { information }\end{array}$ \\
\hline
\end{tabular}

\begin{tabular}{|l|l|l|}
\hline & $\begin{array}{l}\text { Inappropriate } \\
\text { understanding of the } \\
\text { functioning of job }\end{array}$ & $\begin{array}{l}\text { Inappropriately } \\
\text { choosing predictor } \\
\text { variables }\end{array}$ \\
& $\begin{array}{l}\text { Inappropriate } \\
\text { understanding of } \\
\text { required soft skills }\end{array}$ & \\
\hline
\end{tabular}

\section{Conclusion}

Research on AI in management decision-making is growing. However, there seems to be a lack of discussion on cognitive biases in the development process of $\mathrm{AI}$ in the business context. As biases can be self-reinforcing using machine learning, it is critical to investigate the potential biases and errors in AI. In this paper, we proposed a conceptual model that illustrates the roles of managers and developers in developing biased AI recruitment systems.

Managers' past decisions as training datasets in addition to developers' role in choosing and using datasets to train algorithms have been explained. The managers' assumptions and objective functions as well as formulating these assumptions by developers have been discussed.

Based on the collected data, the conceptual model presents some biases in past recruitment decisions and HR managers' assumptions that may lead to biased decisions. Biased decisions happen because of inappropriate understanding of the functioning of job positions and understanding soft skills. Also, missing information in datasets and inappropriate choosing of variables to formulate algorithms are shown as reasons for arising biases in developing AI.

This research contributes to theory and practice. For academics, the conceptual model is an important step in understanding how cognitive biases are involved in AI. Determining the role of managers and developers in developing AI encourages individuals to take this into account when they are providing datasets and formulating algorithms.

Although AI is being used in the business context, academic research about using AI in the business context is rather new. Therefore, there might be some challenges with this study. For example, the researcher may not be allowed to have access to datasets of an organization. Moreover, some managers might not give information about their past biased decisions.

Our proposed model provides insight into the process of AI development for AI-assisted managerial decision-making in the recruitment and selection processes in organizations. Future studies can develop and apply the model to other business contexts such as logistics or marketing. We also suggest that the model be examined by different methodologies such as the 
design science methodology to develop an AI recruitment system and improve the functional performance of AI recruitment systems. This study aims to provide valuable insight and practical guidelines for both managers and developers.

\section{References}

[1] Akerkar, R. (2013). Big Data Computing (R. Akerkar (ed.)). CRC, Taylor \& Francis Group.

[2] Akerkar, R. (2019). Artificial Intelligence for Business. Springer Nature Switzerland AG.

[3] Arnott, D. (2006). Cognitive biases and decision support systems development: a design science approach. Information Systems Journal, 16, 55-78.

[4] Ashford, W. (2018). AI is key to keeping IBM compliant with GDPR. Computer Weekly.

[5] Barnes James. (1984). Cognitive Biases and Their Impact on Strategic Planning. Strategic Management Journal, 5(2), 129-137.

[6] Bazerman, M. H., \& Moore, D. A. (2013). Judgement in Managerial Decision making. Wiley.

[7] Bechmann, A., \& Bowker, G. C. (2019). Unsupervised by any other name: Hidden layers of knowledge production in artificial intelligence on social media. Big Data \& Society, 6(1).

[8] Bonabeau, E. (2003). Trust Your Gut? Don't. Harvard Business Review, 116-123.

[9] Cardwell, M. (2013). Dictionary of Psychology. Chicago IL: Fitzroy Dearborn.

[10] Chirapurath, J. (2019). Knowledge Mining: The Next Wave of Artificial Intelligence-Led Transformation. Harward Business Review Analytic Services.

[11] Colson, E. (2019). What AI-Driven Decision Making Looks Like. Harward Business Review, 1-8.

[12] Cormen, T. (2009). Introduction to algorithms. MIT Press.

[13] Cowgill, B. (2018). Bias and Productivity in Humans and Algorithms: Theory and Evidence from Résumé Screening. Columbia Business School, 29, 1-58.

[14] Deloitte. (2019). How Third-Party Data can Enhance Analytics. CIO Journal. https://deloitte.wsj.com/cio/2019/05/23/how-thirdparty-data-can-enhance-analytics/

[15] Duhaime, I., \& Schwenk, C. (1985). Conjectures on Cognitive Simplification in Acquisition and Divestment Decision Making. Academy of Management Review, 10(2), 287-295.

[16] Edwards, J., \& Rodriguez, E. (2019). Remedies against bias in analytics systems. Journal of Business Analytics, 1-14.

[17] Ehrlinger, J., Readinger, W. O., \& Kim, B. (2016). Decision-Making and Cognitive Biases. In Encyclopedia of Mental Health.

[18] Glaser, B. (1992). Basics of Grounded Theory Analysis: Emergence vs. Forcing. Sociology. Sociology Press.

[19] Glaser, B., \& Strauss, A. (1967). The discovery of grounded theory: Strategies for qualitative research. Aldine De Gruyter.

[20] Howard, J. (2019). Artificial intelligence: Implications for the future of work. American Journal of Industrial Medicine, 62(11), 917-926.

[21] Intezari, A., \& Pauleen, D. (2018). Wisdom, Analytics and Wicked Problems: Integral Decision Making for the Data Age. Routledge.

[22] Jisc. (2014). Using external data. Accessed on: February 29, 2019. [online] https://www.jisc.ac.uk/fullguide/business intelligence

[23] Juliano, C. (2018). Machine learning: What developers and business analysts need to know. InfoWorld. https://www.infoworld.com/article/3259512/machinelearning-what-developers-and-business-analysts-needto-know.html

[24] Kahneman, D., Lovallo, D., \& Sibony, O. (2011). Before you make the big decision. Harvard Business Review, June.

[25] Kaplan, A., \& Haenlein, M. (2019). Rulers of the world, unite! The challenges and opportunities of artificial intelligence. Business Horizons, 63(1), 37-50.

[26] Kaplan, A., \& Haenlein, M. (2019). Siri, Siri, in my hand: Who's the fairest in the land? On the interpretations, illustrations, and implications of artificial intelligence. Business Horizons, 62(1), 1525.

[27] Kleinberg, J., Ludwig, J., Mullainathan, S., \& Sunstein, C. R. (2019). Discrimination in the Age of Algorithms. Journal of Legal Analysis, 2005, 1-62.

[28] Kumar, R. (2017). Machine Learning and Cognition in Enterprises (First). Apress.

[29] Leighton, J. (2010). Cognitive Biases. In A. J. Mills, G. Durepos, \& E. Wiebe (Eds.), Encyclopedia of Case Study Research (pp. 430-434). SAGE Publications.

[30] Manyika, J., Silberg, J., \& Presten, B. (2019). What Do We Do About the Biases in AI? Harvard Business Review, 2-6.

[31] Martin, K. (2018). Ethical Implications and Accountability of Algorithms. Journal of Business Ethics, 1-16.

[32] Minsky, M. (1968). Semantic Information Processing. MIT Press.

[33] Nilsson, N. J. (2010). The Quest for Artificial Intelligence. Cambridge University Press.

[34] Phillips-Wren, G. (2017). Intelligent systems to support human decision making. In Artificial Intelligence: Concepts, Methodologies, Tools, and Applications (Vol. 4, Issue September, pp. 3023-3036).

[35] Russell, S., \& Norvig, P. (2010). Artificial Intelligence A modern Approach third edition. Pearson Education.

[36] Salgado, C., Azevedo, C., Proen, H., \& Vieira, S. (2016). Missing data. In Secondary Analysis of Electronic Health Records (pp. 1-427). Springer.

[37] Schwenk, C. R. (1984). Cognitive simplification processes in strategic decision making. 5(2), 111-128.

[38] Sears, G., \& Rowe, P. (2003). A personality-based similar-to-me effect in the employment interview: Conscientiousness, affect-versus competencemediated interpretations, and the role of job relevance. Canadian Journal of Behavioural Science, 35(1), 1324.

[39] Shrestha, Y. R., Ben-Menahem, S. M., \& von Krogh, G. (2019). Organizational Decision-Making Structures in 
the Age of Artificial Intelligence. California Management Review.

[40] Squire, M. (2015). Clean Data: Save time by discovering effortless strategies for cleaning, organizing, and manipulating your data. Packt.

[41] Tambe, P., Cappelli, P., \& Yakubovich, V. (2019). Artificial Intelligence in Human Resources Management: $\mathrm{C}$ hallenges and a Path Forward . California Management Review.

[42]Taniguchi, H., Sato, H., \& Shirakawa, T. (2018). A machine learning model with human cognitive biases capable of learning from small and biased datasets. In Scientific Reports (Vol. 8, Issue 1).

[43] Varshney, K. (2018). Introducing AI Fairness 360. IBM. https://www.ibm.com/blogs/research/2018/09/aifairness-360/

[44] Zheng, H., Kulkarni, S., \& Poor, V. (2013). A sequential predictor retraining algorithm and its application to market prediction. Annals of Operations Research, 208(1), 209-225. 